Article

\title{
The Application of Generalized Quasi-Hadamard Products of Certain Subclasses of Analytic Functions with Negative and Missing Coefficients
}

\section{En Ao*(1) and Shuhai Li}

School of Mathematics and Statistics, Chifeng University, Chifeng 02400, Inner Mongolia, China

* Correspondence: cfxyaoen@sina.com

Received: 13 June 2019; Accepted: 9 July 2019; Published: 12 July 2019

\begin{abstract}
In this paper, we introduce a new generalized differential operator using a new generalized quasi-Hadamard product, and certain new classes of analytic functions using subordination. We obtain certain results concerning the closure properties of the generalized quasi-Hadamard products and the generalized differential operators for this new subclasses of analytic functions with negative and missing coefficients.
\end{abstract}

Keywords: analytic functions; quasi-Hadamard; differential operator; closure property

MSC: $30 \mathrm{C} 45$

\section{Introduction and Motivation}

Let $\mathcal{A}(a, k)$ denote the class of functions of the form

$$
f(z)=a z+\sum_{n=k}^{\infty} a_{n} z^{n} \quad(a>0, k \in N \backslash\{1\}=\{2,3, \cdots\}),
$$

which are analytic in the unit disk $\mathbb{U}=\{z:|z|<1\}$. Obviously, $\mathcal{A}(1,2)=\mathcal{A}$ denotes the class of functions $f(z)$ normalized by $f(0)=f^{\prime}(0)-1=0$ which are analytic in $\mathbb{U}$.

Set $\mathcal{T}(a, k)$ be the class of functions of the form

$$
f(z)=a z-\sum_{n=k}^{\infty}\left|a_{n}\right| z^{n} \quad(a>0, k \in N \backslash\{1\}=\{2,3, \cdots\}) .
$$

which are analytic in $\mathbb{U}$. It is easy to see that $\mathcal{T}(a, k) \subset \mathcal{A}(a, k)$.

Let $f_{i}(z) \in \mathcal{T}(a, k)(i=1,2)$ be given by

$$
f_{i}(z)=a z-\sum_{n=k}^{\infty}\left|a_{n, i}\right| z^{n}(i=1,2),
$$

then the quasi-Hadamard product (or convolution ) $f_{1} * f_{2}$ is defined by

$$
\left(f_{1} * f_{2}\right)(a ; z)=a^{2} z-\sum_{n=k}^{\infty}\left|a_{n, 1}\right|\left|a_{n, 2}\right| z^{n} .
$$

For any real numbers $p$ and $q$, we define the generalized quasi-Hadamard product $f_{1} \triangle f_{2}$ by

$$
\left(f_{1} \triangle f_{2}\right)(p, q ; a, z)=a^{2} z-\sum_{n=k}^{\infty}\left|a_{n, 1}\right|^{p}\left|a_{n, 2}\right|^{q} z^{n}=\left(f_{2} \triangle f_{1}\right)(p, q ; a, z) .
$$


Clearly, for $p=q=1,\left(f_{1} \triangle f_{2}\right)(1,1 ; a, z)$ reduces to the above quasi-Hadamard product $\left(f_{1} *\right.$ $\left.f_{2}\right)(a ; z)$; for $a=1,\left(f_{1} \triangle f_{2}\right)(p, q ; 1, z)$ reduces to the generalized Hadamard product $\left(f_{1} \triangle f_{2}\right)(p, q ; z)$ defined by Jae Ho Choi and Yong Chan Kim [1]; and for $p=q=1, a=1,\left(f_{1} \triangle f_{2}\right)(1,1 ; 1, z)$ reduces to the quasi-Hadamard product $\left(f_{1} * f_{2}\right)(z)$. For $a=1, p, q \in N \backslash\{1\},\left(f_{1} \triangle f_{2}\right)(p, q ; 1, z)$ reduces to the quasi-Hadamard product $(\underbrace{f_{1} * \cdots f_{1}}_{p} * \underbrace{f_{2} * \cdots f_{2}}_{q})(z)$ (see [2], also see $\left.[3,4]\right)$.

In 1975, Schild and Silverman [5] studied closure properties of the quasi-Hadamard product $\left(f_{1} * f_{2}\right)(z)$ for a starlike function of order $\alpha$ and convex function of order $\alpha$ with negative coefficients in $\mathcal{A}$. In 1983, Owa [2] obtained closure properties of quasi-Hadamard product $\left(f_{1} * f_{2} * \cdots * f_{m}\right)(z)$ and $\left(f_{1} * f_{2} * \cdots * f_{m} * g_{1} * g_{2} \cdots * g_{l}\right)(z)$ for the same function classes in $\mathcal{A}$. Later Kumar [4] improved some results in 1987. In 1992, Srivastava and Owa [6] studied closure properties of quasi-Hadamard product $\left(f_{1} * f_{2} * \cdots * f_{m}\right)(z)$ for $\mathrm{p}$-valent starlike function of order $\alpha$ and p-valent convex function of order $\alpha$ class with negative coefficients in $\mathcal{A}$. In 1996, Jae Ho Choi and Yong Chan Kim [1] introduced the generalized Hadamard product $\left(f_{1} \triangle f_{2}\right)(p, q ; z)$, and obtained the closure properties of $\left(f_{1} \triangle\right.$ $\left.f_{2}\right)(p, q ; z)$ for a starlike function of order $\alpha$ and convex function of order $\alpha$ with negative coefficients in $\mathcal{A}$. Since then, a lot of authors considered and studied closure properties and characteristics of the quasi-Hadamard product $(f * g)(z),\left(f_{1} * f_{2} * \cdots f_{m}\right)(z)$ or $\left(f_{1} * f_{2} * \cdots f_{m} * g_{1} * g_{2} * \cdots g_{l}\right)(z)$ for some classes of normalized analytic functions and normalized meromorphic analytic functions, see, for example, [7-15].

Although the closure properties of Hadamard product or quasi-Hadamard product have already been studied in $\mathcal{A}$, our focus is to introduce generalized quasi-Hadamard product, generalized differential operators, and generalized function classes on non-normalized analytic functions, and to discuss the closure properties on generalized analytic function classes.

Now by using the generalized quasi-Hadamard product $\left(f_{1} \triangle f_{2}\right)(p, q ; a, z)$, we introduce the following differential operator $D^{m}(m \in N)$ as follows:

$$
\begin{gathered}
D^{0}\left(f_{1} \triangle f_{2}\right)=\left(f_{1} \triangle f_{2}\right), \\
D^{1}\left(f_{1} \triangle f_{2}\right)=D\left(f_{1} \triangle f_{2}\right)=z\left(f_{1} \triangle f_{2}\right)^{\prime}, \\
D^{m}\left(f_{1} \triangle f_{2}\right)=D\left(D^{m-1}\left(f_{1} \triangle f_{2}\right)\right) .
\end{gathered}
$$

We define the generalized differential operator $D_{\mu}^{m}(\mu \geq 0)$ as follows:

$$
D_{\mu}^{m}\left(f_{1} \triangle f_{2}\right)=(1-\mu) D^{m}\left(f_{1} \triangle f_{2}\right)+\mu D^{m+1}\left(f_{1} \triangle f_{2}\right) .
$$

If $f_{1} \triangle f_{2}$ is given by (3), then we can obtain that

$$
D^{m}\left(f_{1} \triangle f_{2}\right)(p, q ; a, z)=a^{2} z-\sum_{n=k}^{\infty} n^{m}\left|a_{n, 1}\right|^{p}\left|a_{n, 2}\right|^{q} z^{n}
$$

and

$$
D_{\mu}^{m}\left(f_{1} \triangle f_{2}\right)(p, q ; a, z)=a^{2} z-\sum_{n=k}^{\infty}[1+(n-1) \mu] n^{m}\left|a_{n, 1}\right|^{p}\left|a_{n, 2}\right|^{q} z^{n} .
$$

Clearly, $D_{0}^{m}\left(f_{1} \triangle f_{2}\right)(p, q ; a, z)=D^{m}\left(f_{1} \triangle f_{2}\right), D_{0}^{0}\left(f_{1} \triangle f_{2}\right)(p, q ; a, z)=\left(f_{1} \triangle f_{2}\right)(p, q ; a, z)$. For $a=p=q=1, f_{1}(z)=z-\sum_{n=k}^{\infty}\left|a_{n}\right| z^{n}, f_{2}(z)=\frac{z-z^{2}-z^{k}}{1-z}, D^{m}\left(f_{1} \triangle f_{2}\right)(1,1 ; 1, z)$ becomes Sălăgean operator (see [16]). Also, by specializing the parameters $\mu, p, q$, we obtain the following new operators:

$$
D_{\mu}^{m}\left(f_{1} \triangle f_{2}\right)(1,1 ; a, z)=: D_{\mu}^{m}\left(f_{1} * f_{2}\right)(a ; z)=a^{2} z-\sum_{n=k}^{\infty}[1+(n-1) \mu] n^{m}\left|a_{n, 1}\right|\left|a_{n, 2}\right| z^{n}
$$


and

$$
D_{0}^{m}\left(f_{1} \triangle f_{2}\right)(1,1 ; a, z)=: D^{m}\left(f_{1} * f_{2}\right)(a ; z)=a^{2} z-\sum_{n=k}^{\infty} n^{m}\left|a_{n, 1}\right|\left|a_{n, 2}\right| z^{n} .
$$

For two analytic functions $f$ and $g$, the function $f$ is subordinate to $g$ in $\mathbb{U}$ (see [17]), written as follows

$$
f(z) \prec g(z), z \in \mathbb{U},
$$

if there exists an analytic function $\omega$, with $\omega(0)=0$ and $|\omega(z)|<1$ such that

$$
f(z)=g(\omega(z))
$$

In particular, if the function $g$ is univalent in $\mathbb{U}$, then $f(z) \prec g(z)$ is equivalent to $f(0)=g(0)$ and $f(\mathbb{U}) \subset g(\mathbb{U})$.

We define two generalization classes satisfying the following subordination condition.

Definition 1. $\lambda \geq 0, a>0, A, B \in R,|A| \leq 1,|B| \leq 1, A \neq B$. A function $f(z) \in \mathcal{A}(a, k)$ is in the class $\mathcal{Q}_{\lambda}(a, k, A, B)$ if and only if

$$
(1-\lambda) \frac{f(z)}{z}+\lambda f^{\prime}(z) \prec \frac{a(1+A z)}{1+B z} .
$$

For suitable choices $\lambda, a, k, A, B$, the class $\mathcal{Q}_{\lambda}(a, k, A, B)$ reduces the following subclasses.

$\mathcal{Q}_{\lambda}(a, k, 1-2 \beta,-1)=: \mathcal{Q}_{\lambda}(a, k, \beta)=\left\{f(z) \in \mathcal{A}(a, k):(1-\lambda) \frac{f(z)}{z}+\lambda f^{\prime}(z) \prec \frac{a[1+(1-2 \beta) z]}{1-z}, \beta<1\right\}$. Obviously, $\mathcal{Q}_{\lambda}(1,2, \beta)=: \mathcal{Q}_{\lambda}(\beta)$ (see [18]);

$$
\mathcal{Q}_{\lambda}(1,2, A, B)=: \mathcal{Q}_{\lambda}(A, B)=\left\{f(z) \in \mathcal{A}:(1-\lambda) \frac{f(z)}{z}+\lambda f^{\prime}(z) \prec \frac{1+A z}{1+B z}\right\} ;
$$

$\mathcal{Q}_{0}(a, k, A, B)=: \mathcal{R}(a, k, A, B)=\left\{f(z) \in \mathcal{A}(a, k): \frac{f(z)}{z} \prec \frac{a(1+A z)}{1+B z}\right\} ;$

(4) $\mathcal{Q}_{1}(a, k, A, B)=: \mathcal{H}(a, k, A, B)=\left\{f(z) \in \mathcal{A}(a, k): f^{\prime}(z) \prec \frac{a(1+A z)}{1+B z}\right\}$. Obviously, $\mathcal{H}(1, k, A, B)=$ : $\mathcal{P}_{k}(A, B)=\left\{f(z) \in \mathcal{A}(1, k): f^{\prime}(z) \prec \frac{1+A z}{1+B z},-1 \leq B<A \leq 1\right\}$ (see [19]).

Definition 2. Let $\lambda \geq 0, a>0, A, B \in R,|A| \leq 1,|B| \leq 1, A \neq B$. A function $f(z) \in \mathcal{A}(a, k)$ is in the class $\mathcal{J}_{\lambda}(a, k, A, B)$ if and only if

$$
f^{\prime}(z)+\lambda z f^{\prime \prime}(z) \prec \frac{a(1+A z)}{1+B z} .
$$

Clearly, we have the following equivalence:

$$
f(z) \in \mathcal{J}_{\lambda}(a, k, A, B) \Longleftrightarrow z f^{\prime}(z) \in \mathcal{Q}_{\lambda}(a, k, A, B) .
$$

Let

$$
\begin{aligned}
& \mathcal{T} \mathcal{Q}_{\lambda}(a, k, A, B)=\mathcal{T}(a, k) \bigcap \mathcal{Q}_{\lambda}(a, k, A, B), \\
& \mathcal{T} \mathcal{J}_{\lambda}(a, k, A, B)=\mathcal{T}(a, k) \bigcap \mathcal{J}_{\lambda}(a, k, A, B)
\end{aligned}
$$

Our object of this paper is to the closure properties of the generalized quasi-Hadamard products, the generalized differential operators for the above generalized classes $\mathcal{T} \mathcal{Q}_{\lambda}(a, k, A, B)$ and $\mathcal{T} \mathcal{J}_{\lambda}(a, k, A, B)$. Our results are new in this direction and they give birth to many corollaries.

\section{Preliminary Results}

Due to derive our main result, we need to talk about the following lemmas. 
Lemma 1. $\lambda \geq 0, a>0, A, B \in R,|A| \leq 1,|B| \leq 1, A \neq B$. If the function $f(z)=a z+\sum_{n=k}^{\infty} a_{n} z^{n} \in$ $\mathcal{A}(a, k)$ satisfies

$$
\sum_{n=k}^{\infty}[1+(n-1) \lambda](1+|B|)\left|a_{n}\right| \leq a|A-B|
$$

then $f(z) \in \mathcal{Q}_{\lambda}(a, k, A, B)$.

Proof. We assume that the inequality (5) holds true. According to Definition 1 , the function $f(z) \in$ $\mathcal{Q}_{\lambda}(a, k, A, B)$ if and only if there exists an analytic function $\omega(z), \omega(0)=0,|\omega(z)|<1(z \in \mathbb{U})$ such that

$$
F(z)=\frac{a(1+A \omega(z))}{1+B \omega(z)} \quad(z \in \mathbb{U}),
$$

where

$$
F(z)=(1-\lambda) \frac{f(z)}{z}+\lambda f^{\prime}(z)
$$

or equivalently

$$
\left|\frac{F(z)-a}{a A-B F(z)}\right|<1(z \in \mathbb{U})
$$

it suffices to show that

$$
|F(z)-a|-|a A-B F(z)|<0 .
$$

Therefore, if we let $z \in \partial \mathbb{U}=\{z: z$ is complex number and $|z|=1\}$, we find from (6) that

$$
\begin{aligned}
\mid F(z) & -a|-| a A-B F(z) \mid \\
& =\left|\sum_{n=k}^{\infty}[1+(n-1) \lambda] a_{n} z^{n}\right|-\left|a(A-B)-\sum_{n=k}^{\infty} a(A-B)-\sum_{n=k}^{\infty}[1+(n-1) \lambda] B a_{n} z^{n}\right| \\
& \leq \sum_{n=k}^{\infty}[1+(n-1) \lambda]\left|a_{n}\right||z|^{n}-a|A-B|+\sum_{n=k}^{\infty}[1+(n-1) \lambda]|B|\left|a_{n}\right||z|^{n} \\
& \leq \sum_{n=k}^{\infty}[1+(n-1) \lambda](1+|B|)\left|a_{n}\right|-a|A-B| \leq 0 .
\end{aligned}
$$

Hence, by the maximum modulus theorem, we have $f(z) \in \mathcal{Q}_{\lambda}(a, k, A, B)$. Thus we complete the proof of Lemma 1.

Lemma 2. Let $\lambda \geq 0, a>0$, and the function $f(z)=a z-\sum_{n=k}^{\infty}\left|a_{n}\right| z^{n} \in \mathcal{T}(a, k)$.

$$
\text { If }-1 \leq B<A \leq 1, B \leq 0 \text {, then } f(z) \in \mathcal{T} \mathcal{Q}_{\lambda}(a, k, A, B) \text { if and only if }
$$

$$
\sum_{n=k}^{\infty}[1+(n-1) \lambda](1-B)\left|a_{n}\right| \leq a(A-B) .
$$

$$
\text { If }-1 \leq A<B \leq 1, B \geq 0 \text {, then } f(z) \in \mathcal{T} \mathcal{Q}_{\lambda}(a, k, A, B) \text { if and only if }
$$

$$
\sum_{n=k}^{\infty}[1+(n-1) \lambda](1+B)\left|a_{n}\right| \leq a(B-A) .
$$

The result is sharp for the function $f(z)$ given by

$$
f(z)=a z-\frac{a|A-B|}{[1+(k-1) \lambda](1+|B|)} z^{k} \quad(k \in N \backslash\{1\}=\{2,3, \cdots\}) .
$$

Proof. Since $\mathcal{T} \mathcal{Q}_{\lambda}(a, k, A, B) \subset \mathcal{Q}_{\lambda}(a, k, A, B)$, according to Lemma 1 we only need to prove the 'only if' part of this Lemma. 
Now let us prove the necessity of case (1).

Let $f(z) \in \mathcal{T} \mathcal{Q}_{\lambda}(a, k, A, B),-1 \leq B<A \leq 1, B \leq 0$. Then it satisfies (6) or equivalently

$$
\left|\frac{\sum_{n=k}^{\infty}[1+(n-1) \lambda]\left|a_{n}\right| z^{n-1}}{a(A-B)+\sum_{n=k}^{\infty}[1+(n-1) \lambda] B\left|a_{n}\right| z^{n-1}}\right|<1, z \in \mathbb{U} .
$$

Since $|\Re(z)| \leq|z|, z \in \mathbb{U}$, we have

$$
\Re\left\{\frac{\sum_{n=k}^{\infty}[1+(n-1) \lambda]\left|a_{n}\right| z^{n-1}}{a(A-B)+\sum_{n=k}^{\infty}[1+(n-1) \lambda] B\left|a_{n}\right| z^{n-1}}\right\}<1, z \in \mathbb{U} .
$$

Choose values of $z$ on the real axis so that $(1-\lambda) \frac{f(z)}{z}+\lambda f^{\prime}(z)$ is real. Upon clearing the denominator in (8) and letting $z \rightarrow 1^{-}$through real values, we obtain (7).

Similar to the above proof for case (1), we can prove that case (2) is true. Thus we complete the proof of Lemma 2.

Using arguments similar to those in the proof of Lemmas 1 and 2, we can prove the following Lemmas 3 and 4 .

Lemma 3. Let $\lambda \geq 0, a>0, A, B \in R,|A| \leq 1,|B| \leq 1, A \neq B$. If the function $f(z)=a z+\sum_{n=k}^{\infty} a_{n} z^{n} \in$ $\mathcal{A}(a, k)$ satisfies

$$
\sum_{n=k}^{\infty}[1+(n-1) \lambda](1+|B|)|n| a_{n}|\leq a| A-B \mid,
$$

then $f(z) \in \mathcal{J}_{\lambda}(a, k, A, B)$.

Lemma 4. Let $\lambda \geq 0, a>0$, and the function $f(z)=a z-\sum_{n=k}^{\infty}\left|a_{n}\right| z^{n} \in \mathcal{T}(a, k)$. If $-1 \leq B<A \leq 1, B \leq 0$, then $f(z) \in \mathcal{T} \mathcal{J}_{\lambda}(a, k, A, B)$ if and only if

$$
\sum_{n=k}^{\infty}[1+(n-1) \lambda](1-B) n\left|a_{n}\right| \leq a(A-B) .
$$

$$
\text { If }-1 \leq A<B \leq 1, B \geq 0 \text {, then } f(z) \in \mathcal{T J}_{\lambda}(a, k, A, B) \text { if and only if }
$$

$$
\sum_{n=k}^{\infty}[1+(n-1) \lambda](1+B) n\left|a_{n}\right| \leq a(B-A) .
$$

The result is sharp for the function $f(z)$ given by

$$
f(z)=a z-\frac{a|A-B|}{k[1+(k-1) \lambda](1+|B|)} z^{k} \quad(k \in N \backslash\{1\}=\{2,3, \cdots\}) .
$$

\section{Main Results}

Theorem 1. $p>1$ and the functions $f_{i}(z)(i=1,2)$ defined by (2) belong to $\mathcal{T} \mathcal{Q}_{\lambda}(a, k, A, B)$.

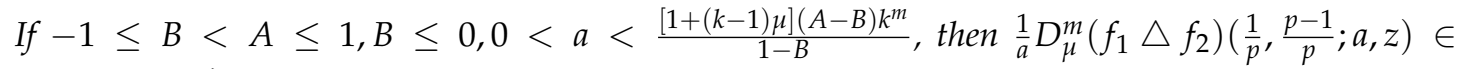
$\mathcal{T} \mathcal{Q}_{\lambda}(a, k, A, \widehat{B})$, where

$$
\frac{a(1-B) A-[1+(k-1) \mu](A-B) k^{m}}{a(1-B)-[1+(k-1)] \mu(A-B) k^{m}} \leq \widehat{B}<\min \{A, 0\} .
$$


If $-1 \leq A<B \leq 1, B \geq 0,0<a<\frac{[1+(k-1) \mu](B-A) k^{m}}{1+B}$, then $\frac{1}{a} D_{\mu}^{m}\left(f_{1} \triangle f_{2}\right)\left(\frac{1}{p}, \frac{p-1}{p} ; a, z\right) \in$ $\mathcal{T} \mathcal{Q}_{\lambda}(a, k, A, \widehat{B})$, where

$$
\max \{A, 0\}<\widehat{B} \leq \frac{a(1+B) A+[1+(k-1) \mu](B-A) k^{m}}{a(1+B)-[1+(k-1) \mu](B-A) k^{m}} .
$$

Proof. (1) Suppose that $-1 \leq B<A \leq 1, B \leq 0$. According to Lemma 2, we need to prove

$$
\sum_{n=k}^{\infty} \frac{[1+(n-1) \lambda](1-\widehat{B})[1+(n-1) \mu]}{a(A-\widehat{B})} \frac{n^{m}}{a}\left|a_{n, 1}\right|^{\frac{1}{p}}\left|a_{n, 2}\right|^{\frac{p-1}{p}} \leq 1 .
$$

Since $f_{i}(z) \in \mathcal{T} \mathcal{Q}_{\lambda}(a, k, A, B)$, by Lemma 2 we have

$$
\left(\sum_{n=k}^{\infty} \frac{[1+(n-1) \lambda](1-B)}{a(A-B)}\left|a_{n, 1}\right|\right)^{\frac{1}{p}} \leq 1
$$

and

$$
\left(\sum_{n=k}^{\infty} \frac{[1+(n-1) \lambda](1-B)}{a(A-B)}\left|a_{n, 2}\right|\right)^{\frac{p-1}{p}} \leq 1 .
$$

By the Hölder inequality we get

$$
\sum_{n=k}^{\infty} \frac{[1+(n-1) \lambda](1-B)}{a(A-B)}\left|a_{n, 1}\right|^{\frac{1}{p}}\left|a_{n, 2}\right|^{\frac{p-1}{p}} \leq 1
$$

Hence the inequality (9) will be satisfied if

$$
\frac{[1+(n-1) \mu](1-\widehat{B}) n^{m}}{a(A-\widehat{B})} \leq \frac{1-B}{A-B}(m, n \in N, n \geq k)
$$

or if

$$
\left[a(1-B)-[1+(n-1) \mu](A-B) n^{m}\right] \widehat{B} \leq a(1-B) A-[1+(n-1) \mu](A-B) n^{m}(m, n \in N, n \geq k) .
$$

Now define the functions $F_{1}(n)$ and $G_{1}(n)$ by

$$
F_{1}(n)=a(1-B)-[1+(n-1) \mu](A-B) n^{m}
$$

and

$$
G_{1}(n)=a(1-B) A-[1+(n-1) \mu](A-B) n^{m} .
$$

When $0<a<\frac{[1+(n-1) \mu](A-B) k^{m}}{1-B}$, we obtain that $F_{1}(n)$ is a decreasing function of $n(n \in N, n \geq k)$ and $F_{1}(n)<F_{1}(k)<0$. Thus the inequality (10) will be satisfied if

$$
\widehat{B} \geq \frac{G_{1}(n)}{F_{1}(n)}=\frac{a(1-B) A-[1+(n-1) \mu](A-B) n^{m}}{a(1-B)-[1+(n-1) \mu](A-B) n^{m}}(m, n \in N, n \geq k) .
$$

We see that the right hand side of (11) is a decreasing function of $n(n \in N, n \geq k)$. Therefore the inequality (10) is satisfied for all $n(n \in N, n \geq k)$ if

$$
\widehat{B} \geq \frac{G_{1}(k)}{F_{1}(k)}=\frac{a(1-B) A-[1+(k-1) \mu](A-B) k^{m}}{a(1-B)-[1+(k-1) \mu](A-B) k^{m}}(m \in N),
$$

which evidently completes the proof of the case (1). 
(2) Suppose that $-1 \leq A<B \leq 1, B \geq 0$. According to Lemma 2, we need to prove

$$
\sum_{n=k}^{\infty} \frac{[1+(n-1) \lambda](1+\widehat{B})[1+(n-1) \mu]}{a(\widehat{B}-A)} \frac{n^{m}}{a}\left|a_{n, 1}\right|^{\frac{1}{p}}\left|a_{n, 2}\right|^{\frac{p-1}{p}} \leq 1 .
$$

Similar to case (1), the inequality (12) will be satisfied if

$$
\frac{[1+(n-1) \mu](1+\widehat{B}) n^{m}}{a(\widehat{B}-A)} \leq \frac{1+B}{B-A}(m, n \in N, n \geq k)
$$

or if

$$
\left[a(1+B)-[1+(n-1) \mu](B-A) n^{m}\right] \widehat{B} \geq a(1+B) A+[1+(n-1) \mu](B-A) n^{m}(m, n \in N, n \geq k)
$$

Now define the functions $F_{2}(n)$ and $G_{2}(n)$ by

$$
F_{2}(n)=a(1+B)-[1+(n-1) \mu](B-A) n^{m}
$$

and

$$
G_{2}(n)=a(1+B) A+[1+(n-1) \mu](B-A) n^{m} .
$$

When $0<a<\frac{[1+(n-1) \mu](B-A) k^{m}}{1+B}$, we obtain that $F_{2}(n)$ is a decreasing function of $n(n \in N, n \geq k)$ and $F_{2}(n)<F_{2}(k)<0$. Thus the inequality (13) will be satisfied if

$$
\widehat{B} \leq \frac{G_{2}(n)}{F_{2}(n)}=\frac{a(1+B) A+[1+(n-1) \mu](B-A) n^{m}}{a(1+B)-[1+(n-1) \mu](B-A) n^{m}}(m, n \in N, n \geq k) .
$$

We see that the right hand side of (14) is an increasing function of $n(n \in N, n \geq k)$. Therefore the inequality (13) is satisfied for all $n(n \in N, n \geq k)$ if

$$
\widehat{B} \leq \frac{G_{2}(k)}{F_{2}(k)}=\frac{a(1+B) A+[1+(n-1) \mu](B-A) k^{m}}{a(1+B)-[1+(n-1) \mu](B-A) k^{m}}(m \in N),
$$

which evidently completes the proof of the case (2). Thus we complete the proof of Theorem 1.

Theorem 2. Let $[1+(n-1) \mu]|A-B| n^{m} \leq[1+(n-1) \lambda](1+|B|)$. If the functions $f_{i}(z)(i=1,2)$ defined by (2) belong to $\mathcal{T} \mathcal{Q}_{\lambda}(a, k, A, B)$, then $\frac{1}{a} D_{\mu}^{m}\left(f_{1} * f_{2}\right)(a ; z) \in \mathcal{T} \mathcal{Q}_{\lambda}(a, k, A, B)$.

Proof. Suppose that $-1 \leq B<A \leq 1, B \leq 0$. According to Lemma 2, we need to prove

$$
\sum_{n=k}^{\infty}[1+(n-1) \lambda](1-B)[1+(n-1) \mu] \frac{n^{m}}{a}\left|a_{n, 1}\right|\left|a_{n, 2}\right| \leq a(A-B) .
$$

Since $f_{i}(z) \in \mathcal{T} \mathcal{Q}_{\lambda}(a, k, A, B)(i=1,2)$, by using Lemma 2 we get

$$
\sum_{n=k}^{\infty}[1+(n-1) \lambda](1-B)\left|a_{n, 1}\right| \leq a(A-B)
$$

and

$$
\sum_{n=k}^{\infty}[1+(n-1) \lambda](1-B)\left|a_{n, 2}\right| \leq a(A-B) .
$$


Therefore, by the Cauchy-Schwarz inequality, we obtain

$$
\sum_{n=k}^{\infty}[1+(n-1) \lambda](1-B) \sqrt{\left|a_{n, 1}\right|\left|a_{n, 2}\right|} \leq a(A-B) .
$$

This implies that we only need to show that

$$
[1+(n-1) \lambda](1-B)[1+(n-1) \mu] \frac{n^{m}}{a}\left|a_{n, 1}\right|\left|a_{n, 2}\right| \leq[1+(n-1) \lambda](1-B) \sqrt{\left|a_{n, 1}\right|\left|a_{n, 2}\right|}(n \geq k)
$$

or, equivalently, that

$$
\sqrt{\left|a_{n, 1}\right|\left|a_{n, 2}\right|} \leq \frac{a}{[1+(n-1) \mu] n^{m}}(n \geq k) .
$$

From (18), the inequality (19) is satisfied for all $n(n \in N, n \geq k)$ if

$$
[1+(n-1) \mu](A-B) n^{m} \leq[1+(n-1) \lambda](1-B)(n \geq k) .
$$

Based on the given condition, we get (15).

Also applying Lemma 2 we can prove $\frac{1}{a} D_{\mu}^{m}\left(f_{1} * f_{2}\right)(a ; z) \in \mathcal{T} \mathcal{Q}_{\lambda}(a, k, A, B)$ for $-1 \leq A<B \leq 1$, $B \geq 0$. Thus we complete the proof of Theorem 2 .

Remark 1. (1) Setting $\mu=0$ in Theorem 1 , we can obtain the closure properties of $\frac{1}{a} \widetilde{D}^{m}\left(f_{1} * f_{2}\right)\left(\frac{1}{p}, \frac{p-1}{p} ; a, z\right)$ for $\mathcal{T} \mathcal{Q}_{\lambda}(a, k, A, B) ;(2)$ Setting $\mu=0$ in Theorem 2, we can obtain the closure properties of $\frac{1}{a} \widetilde{D}_{\mu}^{m}\left(f_{1} * f_{2}\right)(a ; z)$ for $\mathcal{T} \mathcal{Q}_{\lambda}(a, k, A, B)$.

Example 1. Let $p>1,-1 \leq B<A \leq 1, B \leq 0,0<a<\frac{A-B}{1-B}$. If the functions $f_{i}(z)(i=1,2)$ defined by (2) belong to $\mathcal{T} \mathcal{Q}_{\lambda}(a, k, A, B)$, then $\frac{1}{a}\left(f_{1} \triangle f_{2}\right)\left(\frac{1}{p}, \frac{p-1}{p} ; a, z\right) \in \mathcal{T} \mathcal{Q}_{\lambda}(a, k, A, \widehat{B})$, where

$$
\frac{a(1-B) A-(A-B)}{a(1-B)-(A-B)} \leq \widehat{B}<\min \{A, 0\}
$$

\section{Corollaries and Consequences}

On the one hand, by taking special values of parameters $A, B, \lambda, a, k$ we easily obtain the following closure properties for some important subclasses in $\mathcal{A}(a, k)$.

Putting $A=1-2 \beta(0 \leq \beta<1), B=-1$, we obtain the closure properties for the subclass

$$
\mathcal{T} \mathcal{Q}_{\lambda}(a, k, \beta)=\mathcal{T}(a, k) \bigcap \mathcal{Q}_{\lambda}(a, k, \beta)=\left\{f(z) \in \mathcal{T}(a, k):(1-\lambda) \frac{f(z)}{z}+\lambda f^{\prime}(z) \prec \frac{a[1+(1-2 \beta) z]}{1-z}\right\} .
$$

Corollary 1. $p>1,0<\beta<1$ and the functions $f_{i}(z)(i=1,2)$ defined by (2) belong to $\mathcal{T} \mathcal{Q}_{\lambda}(a, k, \beta)$. If $0<a<[1+(k-1) \mu](1-\beta) k^{m}$, then $\frac{1}{a} D_{\mu}^{m}\left(f_{1} \triangle f_{2}\right)\left(\frac{1}{p}, \frac{p-1}{p} ; a, z\right) \in \mathcal{T} \mathcal{Q}_{\lambda}(a, k, 1-2 \beta, \widehat{B})$, where

$$
\frac{a(1-\beta)-[1+(k-1) \mu](1-\beta) k^{m}}{a-[1+(k-1) \mu](1-\beta) k^{m}} \leq \widehat{B}<\min \{1-2 \beta, 0\} .
$$

Corollary 2. Let $0<\beta<1,[1+(n-1) \mu](1-\beta) n^{m} \leq[1+(n-1) \lambda]$. If the functions $f_{i}(z)(i=1,2)$ defined by (2) belong to $\mathcal{T} \mathcal{Q}_{\lambda}(a, k, \beta)$, then $\frac{1}{a} D_{\mu}^{m}\left(f_{1} * f_{2}\right)(a ; z) \in \mathcal{T} \mathcal{Q}_{\lambda}(a, k, \beta)$. Putting $\lambda=0$ and $\lambda=1$, we obtain the closure properties for the subclasses

$$
\mathcal{T} \mathcal{R}(a, k, A, B)=\mathcal{T}(a, k) \bigcap \mathcal{H}(a, k, A, B)=\left\{f(z) \in \mathcal{T}(a, k): \frac{f(z)}{z} \prec \frac{a(1+A z)}{1+B z}\right\}
$$


and

$$
\mathcal{T} \mathcal{H}(a, k, A, B)=\mathcal{T}(a, k) \bigcap \mathcal{H}(a, k, A, B)=\left\{f(z) \in \mathcal{T}(a, k): f^{\prime}(z) \prec \frac{a(1+A z)}{1+B z}\right\}
$$

Corollary 3. Let $[1+(n-1) \mu]|A-B| n^{m} \leq(1+|B|)$. If the functions $f_{i}(z)(i=1,2)$ defined by (2) belong to $\mathcal{T} \mathcal{R}(a, k, A, B)$, then $\frac{1}{a} D_{\mu}^{m}\left(f_{1} * f_{2}\right)(a ; z) \in \mathcal{T} \mathcal{R}(a, k, A, B)$.

Corollary 4. Let $[1+(n-1) \mu]|A-B| n^{m} \leq n(1+|B|)$. If the functions $f_{i}(z)(i=1,2)$ defined by (2) belong to $\mathcal{T H}(a, k, A, B)$, then $\frac{1}{a} D_{\mu}^{m}\left(f_{1} * f_{2}\right)(a ; z) \in \mathcal{T H}(a, k, A, B)$. Putting $a=1, k=2$, we obtain the closure properties for the subclass

$$
\mathcal{T} \mathcal{Q}_{\lambda}(A, B)=\mathcal{T}(1,2) \bigcap \mathcal{Q}_{\lambda}(A, B)=\left\{f(z) \in \mathcal{T}(1,2):(1-\lambda) \frac{f(z)}{z}+\lambda f^{\prime}(z) \prec \frac{a(1+A z)}{1+B z}\right\} .
$$

Corollary 5. Let $p>1$ and the functions $f_{i}(z)(i=1,2)$ defined by (2) belong to $\mathcal{T} \mathcal{Q}_{\lambda}(A, B)$. If $-1 \leq B<$ $A \leq 1, B \leq 0,(1+\mu)(A-B) 2^{m}-(1-B)>0$, then $D_{\mu}^{m}\left(f_{1} \triangle f_{2}\right)\left(\frac{1}{p}, \frac{p-1}{p} ; 1, z\right) \in \mathcal{T} \mathcal{Q}_{\lambda}(A, \widehat{B})$, where

$$
\frac{a(1-B) A-(1+\mu)(A-B) 2^{m}}{a(1-B)-(1+\mu)(A-B) 2^{m}} \leq \widehat{B}<\min \{A, 0\}
$$

Corollary 6. Let $[1+(n-1) \mu]|A-B| 2^{m} \leq[1+(n-1) \lambda](1+|B|)$. If the functions $f_{i}(z)(i=1,2)$ defined by (2) belong to $\mathcal{T} \mathcal{Q}_{\lambda}(A, B)$, then $\frac{1}{a} D_{\mu}^{m}\left(f_{1} * f_{2}\right)(a ; z) \in \mathcal{T} \mathcal{Q}_{\lambda}(A, B)$.

Example 2. Let $p>1,0<\beta<1$. If $f_{i}(z)=z-\sum_{n=2}^{\infty}\left|a_{n, i}\right| z^{n} \in \mathcal{T} \mathcal{Q}_{\lambda}(1-2 \beta,-1), i=1,2$, then $\left(f_{1} \triangle f_{2}\right)\left(\frac{1}{p}, \frac{p-1}{p} ; 1, z\right) \in \mathcal{T} \mathcal{Q}_{\lambda}(1-2 \beta, \widehat{B})$, where $-1 \leq \widehat{B}<\min \{1-2 \beta, 0\}$.

On the other hand, we can obtain the following closure properties for $\mathcal{T} \mathcal{J}(a, k, A, B)$ according to (4) and Lemma 4.

Corollary 7. Let $p>1$ and the functions $f_{i}(z)(i=1,2)$ defined by (2) belong to $\mathcal{T} \mathcal{J}_{\lambda}(a, k, A, B)$.

(1) If $-1 \leq B<A \leq 1, B \leq 0,0<a<\frac{[1+(k-1) \mu](A-B) k^{m}}{1-B}$, then $\frac{1}{a} D_{\mu}^{m}\left(f_{1} \triangle f_{2}\right)\left(\frac{1}{p}, \frac{p-1}{p} ; a, z\right) \in$ $\mathcal{T}_{\lambda}(a, k, A, \widehat{B})$, where

$$
\frac{a(1-B) A-[1+(k-1) \mu](A-B) k^{m}}{a(1-B)-[1+(k-1)] \mu(A-B) k^{m}} \leq \widehat{B}<\min \{A, 0\} .
$$

(2) If $-1 \leq A<B \leq 1, B \geq 0,0<a<\frac{[1+(k-1) \mu](B-A) k^{m}}{1+B}$, then $\frac{1}{a} D_{\mu}^{m}\left(f_{1} \triangle f_{2}\right)\left(\frac{1}{p}, \frac{p-1}{p} ; a, z\right) \in$ $\mathcal{T} \mathcal{J}_{\lambda}(a, k, A, \widehat{B})$, where

$$
\max \{A, 0\}<\widehat{B} \leq \frac{a(1+B) A+[1+(k-1) \mu](B-A) k^{m}}{a(1+B)-[1+(k-1) \mu](B-A) k^{m}} .
$$

Corollary 8. Let $[1+(n-1) \mu]|A-B| n^{m-1} \leq[1+(n-1) \lambda](1+|B|)$. If the functions $f_{i}(z)(i=1,2)$ defined by (2) belong to $\mathcal{T} \mathcal{J}_{\lambda}(a, k, A, B)$, then $\frac{1}{a} D_{\mu}^{m}\left(f_{1} * f_{2}\right)(a ; z) \in \mathcal{T}_{\lambda}(a, k, A, B)$.

\section{Conclusions}

In this paper, we mainly study the closure properties of the generalized quasi-Hadamard products, the generalized differential operator and its related special operators for $\mathcal{T} \mathcal{Q}_{\lambda}(a, k, A, B)$ and $\mathcal{T} \mathcal{J}_{\lambda}(a, k, A, B)$ of analytic functions with negative and missing coefficients. Also, we give two examples and six corollaries to illustrate our results obtained. In the future, we can consider to extend some classical analytic function classes (such as starlike, convex, close-to-convex) in $\mathcal{A}(a, k)$, and discuss the closure properties of the generalized quasi-Hadamard products. 
Author Contributions: Conceptualization, E.A. and S.L.; methodology, E.A. and S.L.; software, E.A.; validation, E.A.; formal analysis, S.L.; investigation, E.A.; resources, S.L.; data curation, E.A.; writing一original draft preparation, E.A.; writing-review and editing, E.A.; visualization, S.L.; supervision, E.A.; project administration, E.A.; funding acquisition, E.A.

Funding: This study was supported by the Higher School Foundation of Inner Mongolia of China(Grant No.NJZY19211) and the National Natural Science Foundation of China(Grant No.11561001).

Conflicts of Interest: The authors declare no conflict of interest.

\section{References}

1. Choi, J.H.; Kim, Y.C.; Owa, S. Generalizations of Hadamard products of functions with negative coefficients. J. Math. Anal. Appl. 1996, 199, 495-501. [CrossRef]

2. Owa, S. On the Hadamard products of univalent functions. Tamkang J. Math. 1983, 14, 15-21.

3. Kumar, V. Hadamard product of certain starlike functions. J. Math. Anal. Appl. 1985, 110, 425-428. [CrossRef]

4. Kumar, V. Quasi-Hadamard product of certain univalent functions. J. Math. Anal. Appl. 1987, 126, 70-77. [CrossRef]

5. Schild, A.; Silveman, H. Convluctions of univalent functions with negative coefficients. Ann. Univ. Mariea Curie-Sklodowsca Sect. A 1975, 29, 99-107.

6. Srivasta, H.M.; Owa, S. Current Topics in Analytic Function Theory; World Scientific Publishing Co., Pte. Ltd.: Singapore, 1992; pp. 234-251.

7. Aouf, M.K. The quasi-Hadamard product of certain analytic functions. Appl. Math. Lett. 2008, 21, 1184-1187. [CrossRef]

8. Frasin, B.A. Quasi-Hadamard product of certain classes of uniformly analytic functions. Gen. Math. 2007, 16, 29-35.

9. Darwish, H.E. The quasi-Hadamard product of certain starlike and convex functions. Appl. Math. Lett. 2007, 20, 692-695. [CrossRef]

10. Frasin, B.A.; Aouf, M.K. Quasi-Hadamard product of certain merpmorphic p-valent analytic functions. Appl. Math. Lett. 2010, 23, 347-350. [CrossRef]

11. Goyal, S.P.; Goswami, P. Quasi-Hadamard product of a generalized class of analytic and univalent functions. Eur. J. Pure Appl. Math. 2010, 6, 1118-1123.

12. El-Ashwah, R.M.; Aouf, M.K.; Breaz, N. On quasi-Hadamard products of p-valent functions with negative coefficients define by using a differential operator. Acta Univ. Apulensis 2011, 28, 61-70.

13. Breaz, N.; El-Ashwah, R.M. Quasi-Hadamard product of some uniformly analytic and p-valent functions with negative coefficients. Carpathian J. Math. 2014, 30, 39-45.

14. EL-Ashwaha, R.M.; Drbuk, M.E. On certain properties for Hadamard product of uniformly univalent meromorphic functions with positive coefficients. Theory Appl. Math. Comput. Sci. 2016, 6,13-18.

15. Bulut, S. The Quasi-Hadamard Product of Certain Analytic and P-Val. Functions. J. Math. 2017, 15, 777-781.

16. Sǎlăgean, G.S. Subclasses of univalent functions. In complex analysis-fifth romanian-finnish seminar, part I (Bucharest, 1981). Lect. Notes Math 1983, 1013, 362-372.

17. Littlewood, J.E. Lectures on the Theory of Functions; Oxford University Press: Oxford, UK, 1944.

18. Ding, S.S.; Ling, Y.; Bao, G.J. Some properties of a class of analytic functions. J. Math. Anal. Appl. 1995, 195, 71-81. [CrossRef]

19. Kumar, V. On univalent functions with negative and missing coefficients. J. Math. Res. Expos. 1984, 4, 27-34. 